\title{
Sinonasal Rosai-Dorfman Disease
}

\author{
${ }^{1}$ Kiran Natarajan, ${ }^{2}$ Amarnath Devarasetty, ${ }^{3}$ Sathiya Murali, ${ }^{4}$ A Senthilvadivu, ${ }^{5}$ Sudhamaheswari, ${ }^{6}$ Mohan Kameswaran
}

\section{ABSTRACT}

Aim: Rosai-dorfman disease (RDD) is a rare histiocytic proliferative disorder characterized by painless massive lymphadenopathy. Extranodal manifestations may occur in the head and neck area. This is a case report of a patient with sinonasal RDD and its management.

Materials and methods: This is a case report of a 36-yearold patient with sinonasal RDD. The clinical features, imaging and histological features are described. The patient underwent endoscopic surgery. Recurrence (8 months later) was managed by laserization of the nasal mass with KTP/532 laser followed by oral corticosteroid therapy.

Results: A 36-year-old lady presented with complaints of left sided nasal obstruction, nasal discharge and left facial discomfort of 6 months duration. There was history of occasional blood stained nasal discharge. Diagnostic nasal endoscopy revealed a polypoidal mass in the left nasal cavity. CT scans revealed soft tissue opacity in the left maxillary antrum, left ostiomeatal complex and extending into the left orbit. There was erosion of the posterolateral wall of the left maxilla. The patient underwent endoscopic surgery and had an uneventful postoperative recovery. Biopsy was reported as Rosai-Dorfman disease. Subsequently, 8 months later, the patient reported with a complaint of nasal obstruction and discharge. A recurrent lesion was noted in the left nasal cavity and maxillary antrum. The mass was managed by laserization followed by oral corticosteroid therapy. One year later, the patient is free from disease and has been advised regarding the necessity of periodic follow-ups.

Conclusion: RDD is a rare disease encountered by the otolaryngologist. The manifestation of extranodal RosaiDorfman disease is most commonly seen in the nasal cavity and paranasal sinuses. The clinical findings and imaging characteristics are variable and the diagnosis can be easily missed. Otolaryngologists and pathologists must be aware of the clinical presentation, imaging characteristics and histologic features of Rosai-Dorfman disease.

Keywords: Sinonasal Rosai-Dorfman disease, Emperipolesis, S100 protein.

\footnotetext{
${ }^{1,3-6}$ Consultant Surgeon, ${ }^{2}$ Postgraduate

${ }^{1,3-6}$ Department of ENT, Madras ENT Research Foundation Chennai, Tamil Nadu, India

${ }^{2}$ Department of Otolaryngology, Madras ENT Research Foundation, Chennai, Tamil Nadu, India

Corresponding Author: Kiran Natarajan, Consultant Surgeon Department of ENT, 1st Cross Street, Off 2nd Main Road, Raja Annamalai Puram, Chennai-600041, Tamil Nadu, India, Phone: 044-2431141115, e-mail: kirannatarajan2001@yahoo.co.in
}

How to cite this article: Natarajan K, Devarasetty A, Murali S, Senthilvadivu A, Sudhamaheswari, Kameswaran M. Sinonasal Rosai-Dorfman Disease. Clin Rhinol An Int J 2014;7(3):142-146.

\section{Source of support: Nil}

\section{Conflict of interest: None}

\section{INTRODUCTION}

Rosai-Dorfman disease (RDD) or syndrome (RDS) is a rare disorder characterized by proliferation and accumulation of histiocytes, usually manifesting as massive enlargement of cervical lymph nodes. Isolated extranodal form presents in 25 to $40 \%$ of cases. ${ }^{1}$ The clinical course of the disease is variable. Approximately 15\% of RDD patients have ear, nose and throat involvement. ${ }^{2}$ The commonest ENT manifestation is in the nose and paranasal sinuses although various other sites in the head and neck could be affected. In sinonasal RDD, patients present with progressive nasal obstruction, nasal discharge, epistaxis, facial pain, hyposmia or anosmia and may also present with pharyngitis and tonsillitis. The diagnosis of RDS is made on the basis of clinical suspicion and confirmed by histopathology and immunohistochemistry. Histiocytes contain intact appearing lymphocytes and less often, plasma cells, neutrophils and red blood cells. This phenomenon of lymphocytes inside the cytoplasm of histiocytes is known as emperipolesis. ${ }^{3}$ This is characteristic of Rosai-Dorfman disease. However, this is found much less commonly in extranodal lesions. The clinical and histopathologic features of this disease may be overlooked. We report a case of sinonasal RDD, the clinical presentation, diagnosis and management.

\section{CASE REPORT}

A 36-year-old lady presented with complaints of left sided progressive nasal obstruction, nasal discharge, left facial discomfort and occasional blood stained nasal discharge of 6 months duration. She had an otherwise unremarkable medical history. Anterior rhinoscopy revealed a reddish polypoidal left nasal mass. The remainder of the ENT examination was unremarkable. Diagnostic nasal endoscopy revealed a polypoidal mass in the left nasal cavity (Fig. 1). She did not have cervical adenopathy or any evidence of systemic illness. CT scans revealed soft tissue opacity in the left maxillary antrum, left ostiomeatal complex and extending into the left orbit 
(Figs 2 and 4). There was erosion of the posterolateral wall of the left maxilla (Fig. 3). Hematological and biochemistry investigations were normal. The patient underwent endoscopic surgery and no unusual bleeding occurred from the lesion during surgery (Fig. 5). However, the mass had caused erosion of the posterior wall of the maxilla. The patient had an uneventful postoperative recovery. Histopathology showed polypoid edematous connective tissue covered by respiratory epithelium with subepithelial infiltrates of lymphocytes, plasma cells and focally some foam cells, few eosinophils and mast cells. Large histiocytes with aggregates of lymphocytes and nuclear debris in the cytoplasm (emperipolesis) was seen which is characteristic for Rosai-Dorfman disease (Fig. 6). Immunohistochemistry was done which was reported as S100 and CD 68 positive (histiocytic cells), LCA and CD1a negative. As Rosai-Dorfman disease is a benign condition, no further treatment was given. The patient was advised regular follow-ups. Subsequently, 8 months later, the patient again reported of left

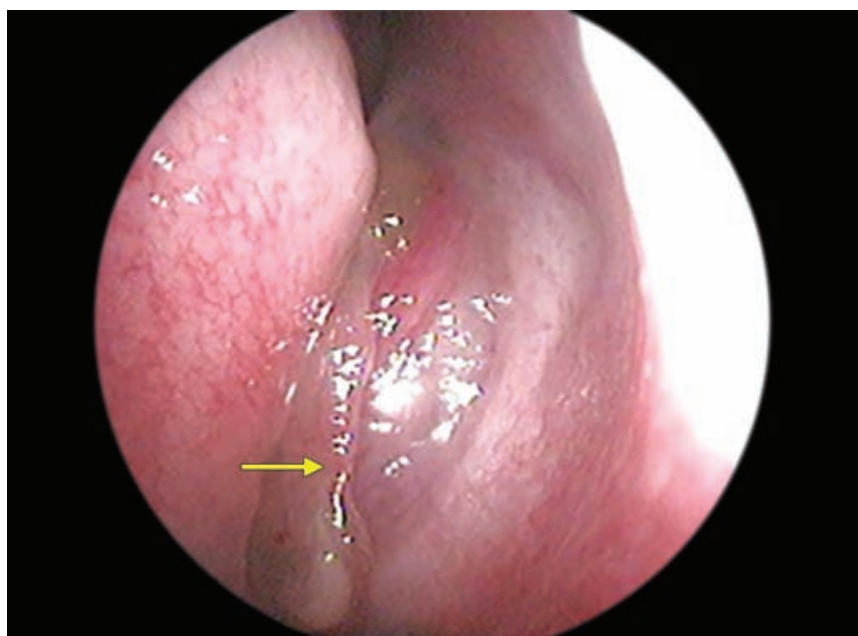

Fig. 1: Diagnostic nasal endoscopy showing polypoid lesion in the left nasal cavity

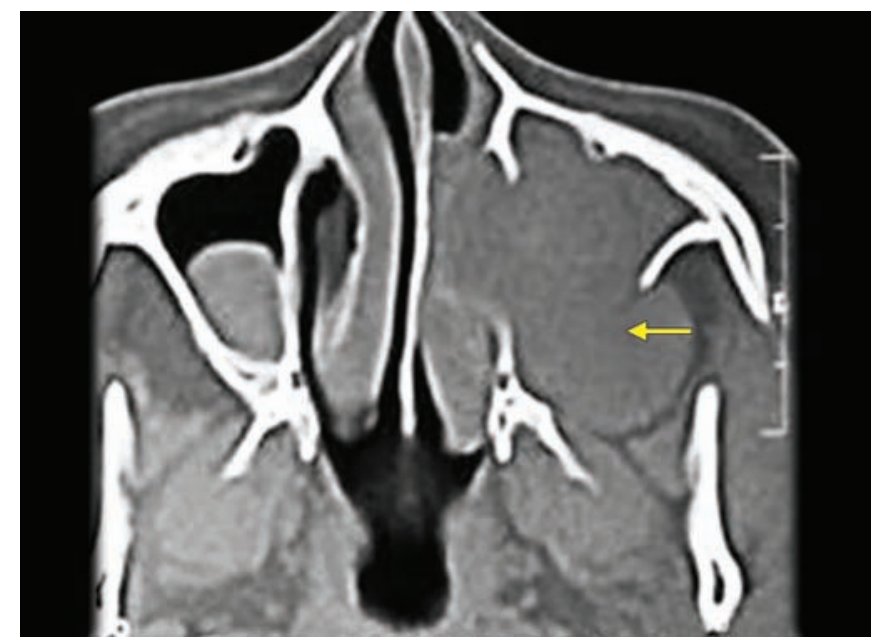

Fig. 3: Erosion of the posterior wall of maxilla by the mass sided nasal obstruction and rhinorrhea and a recurrent lesion was noted in the left nasal cavity and maxillary antrum seen through the previous antrostomy (Fig. 7). No cervical lymphadenopathy was present. The mass was excised by laserization with KTP/532 laser (Fig. 8) followed by oral corticosteroid therapy (60 mg/day for 1 week and then tapered over the next 3 weeks) because of the recurrence. Postoperative diagnostic nasal endoscopy was unremarkable (Fig. 9). One year later, the patient is free from disease and is under regular follow-up. The need for long-term follow-up was emphasized to the patient.

\section{DISCUSSION}

Rosai-Dorfman disease or 'sinus histiocytosis with massive lymphadenopathy' was initially described in the French literature as a lipid storage disorder (adénites avec surcharge lipidique) possibly developing after inflammation. ${ }^{4}$ Rosai-Dorfman disease or syndrome (RDD or RDS) has been named after two pathologists, Ronald F

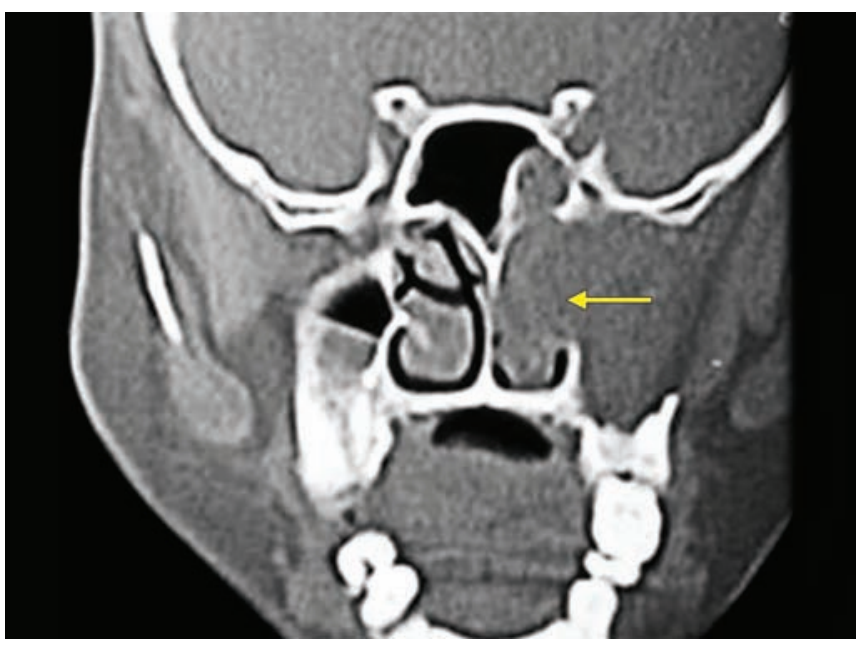

Fig. 2: CT scans of the paranasal sinuses showing soft tissue opacity involving the left maxillary antrum, left nasal cavity, with erosion of the lateral wall of the maxilla

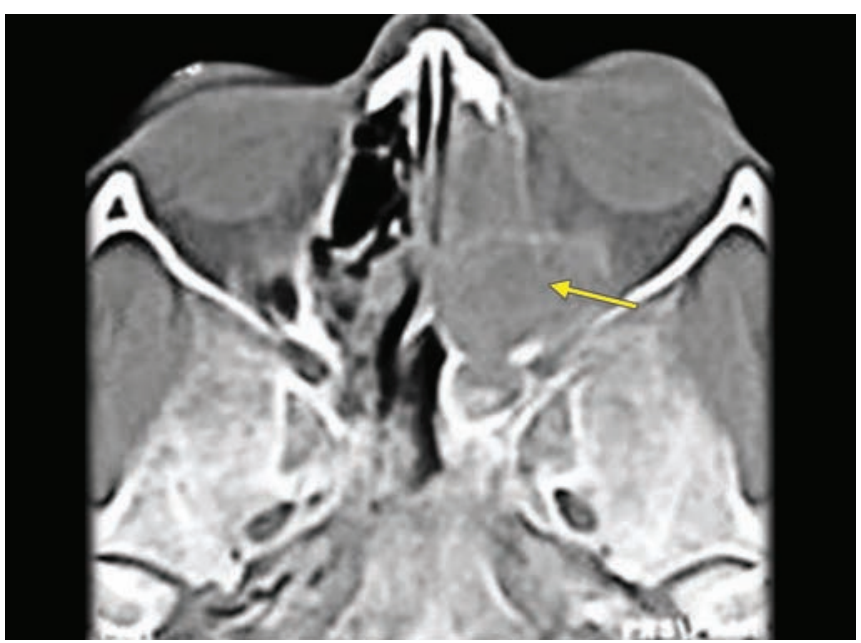

Fig. 4: Orbital encroachment by the lesion 


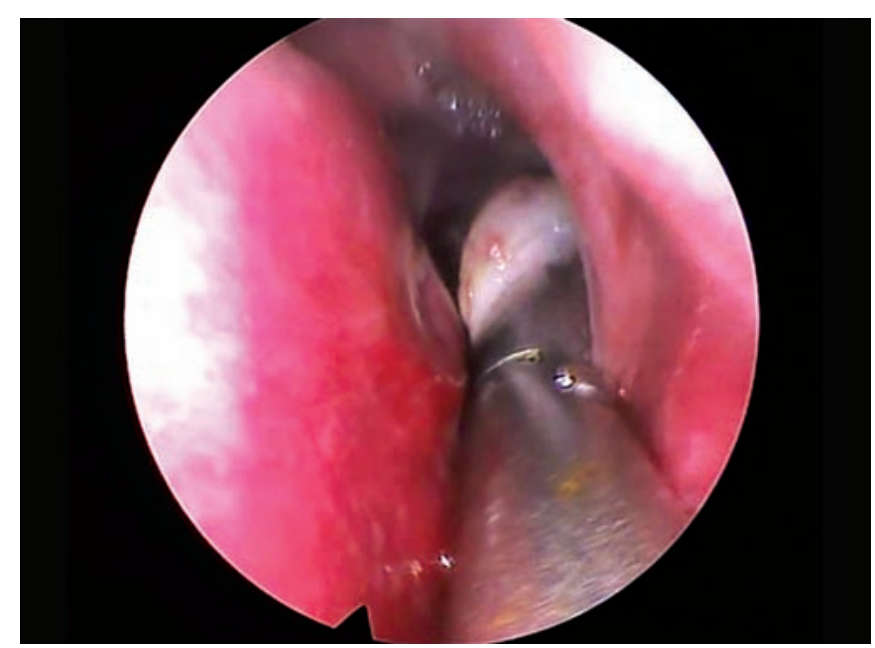

Fig. 5: Endoscopic removal of left sinonasal lesion

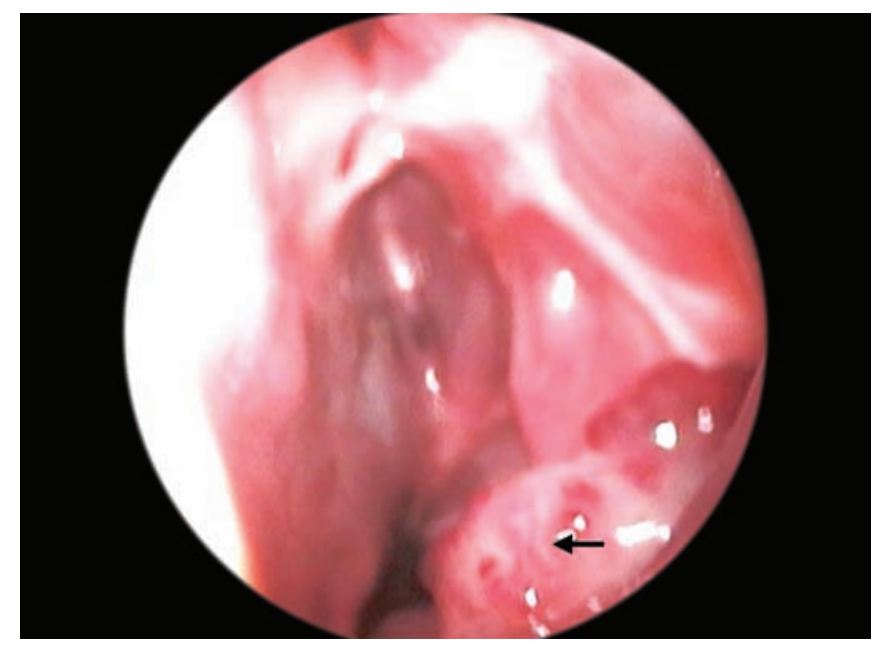

Fig. 7: DNE showing recurrent left sinonasal lesion

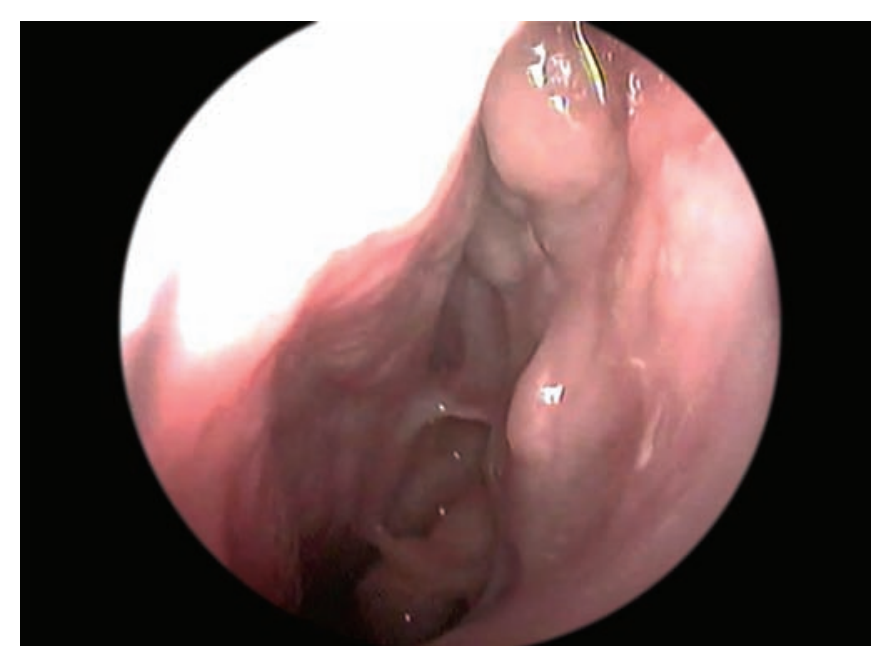

Fig. 9: Postoperative DNE picture showing left nasal cavity free of disease

Dorfman and Juan Rosai in 1969. An alternative eponym of this condition is Destombes-Rosai-Dorfman syndrome. RDS is a non-Langerhans histiocytic disease. It is a benign condition, usually manifesting as massive enlargement

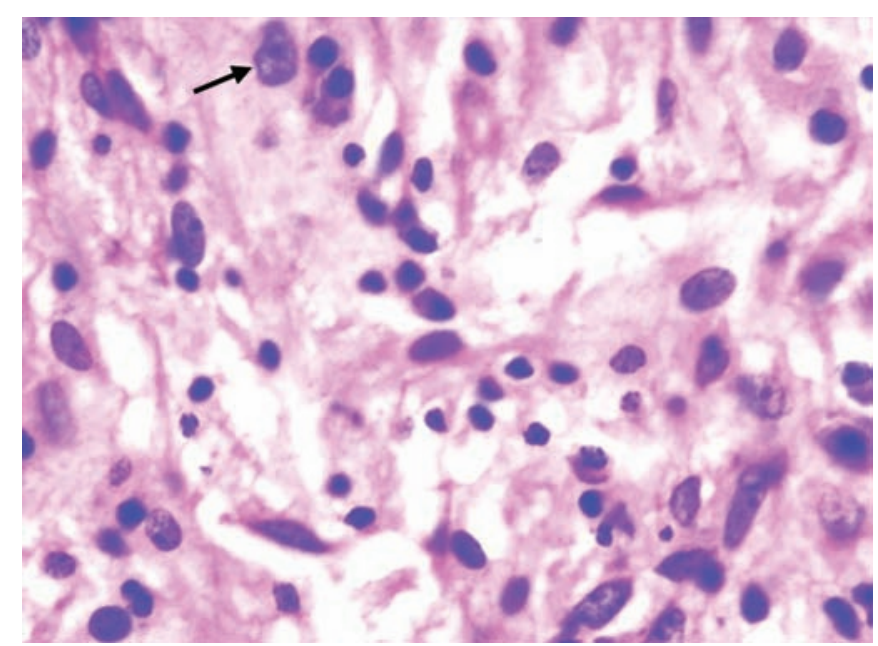

Fig. 6: Histopathology picture of Rosai-Dorfman disease showing emperipolesis (Hematoxylin and Eosin Stain, 40x magnification)

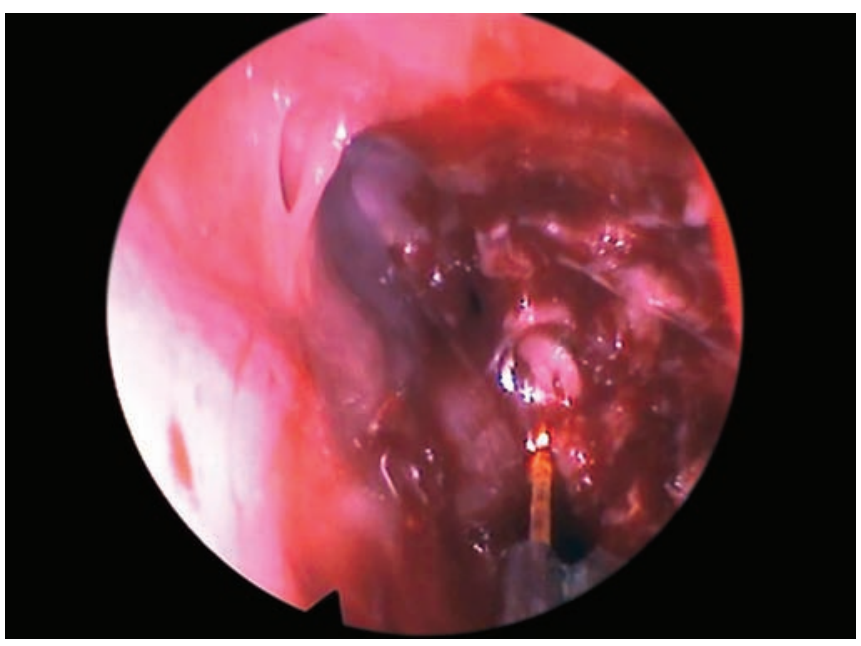

Fig. 8: Intraoperative picture showing laserization of left nasal mass with $\mathrm{KTP} / 532$ laser

of cervical lymph nodes, usually painless and bilateral. ${ }^{5}$ Lymph nodes are isolated, mobile, and small during the initial stages but become adherent with disease progression, forming a voluminous multinodular mass. ${ }^{6}$ Fever, anemia, neutrophilia, leukocytosis, an increased erythrocyte sedimentation rate, and hypergammaglobulinemia may occur. ${ }^{7}$ Other lymphatic groups, such as mediastinal, axillary and inguinal lymph nodes can also be affected, but always to a lesser extent than cervical involvement. Episodes of exacerbation and remissions may occur. Extranodal involvement can occur in eyelids, orbit, respiratory tract, salivary glands, skin, bone, testis, lung, kidney, central nervous system, liver, spleen, spinal cord, thyroid, pancreas and gastrointestinal tract. The most common sites of extranodal involvement are skin followed by nasal and paranasal cavities, subcutaneous tissue, orbit, eyelids and bone. ${ }^{8}$ In patients with extranodal disease, approximately $75 \%$ occur in the head and neck region. ${ }^{9}$ Autoimmune hemolytic anemia can rarely occur. RDS can rarely present with massive hemoptysis. 
The most frequent otorhinolaryngologic manifestations are found in the nasal cavity and paranasal sinuses. Other sites include oral cavity, pharynx, tonsils, maxillary bone, sphenoid bone, frontal bone, skull base, salivary glands, external or middle ear and trachea.

The cause of RDD has yet to be established, but is thought to be an aberrant response to an unspecified antigen, possibly an infective agent or an aberrant response of macrophages to cytokines. Infectious agents that have been studied in association with RDD include Epstein Barr Virus (EBV) and Human Herpes Virus 6 (HHV-6). ${ }^{10}$ A genetic etiology was proposed by Sertac and Rossbach due to the existence of familial cases of RDD. ${ }^{11}$ The clinical course of the disease is variable. The disease shows a worldwide distribution and can manifest in any age group. Occurrence in the first and second decades of life is more common. RDS has a 2:1 male-to-female ratio.

In majority of cases, patients with RDS are in good general health without significant symptoms of disease. When the nasal cavities and paranasal sinuses are involved, patients often present with progressive nasal obstruction but may also present with facial pain, epistaxis, hyposmia or anosmia. Intermittent episodes of spontaneous nasal bleed, which settles on its own have been reported. The most common examination finding in the case of nasal involvement is 'polyposis', where the polyps are coarse and reddish yellow-brown; the typical edematous, glass-like appearance is not found in this disease. ${ }^{10}$

The diagnosis of RDS is made on the basis of clinical suspicion, imaging and confirmed by histopathology and immunohistochemical studies. CT and MRI findings are nonspecific and typically reveal polypoid masses, mucosal thickening, or soft-tissue opacification of the paranasal sinuses or nasal cavity, with or without associated osseous erosion. MRI is superior to CT in the delineation of the lesion, extent and in the discrimination of tumor invasion from the obstructive sinus secretions. It is slightly hyperintense to the muscle on T1-weighted MR images, with intermediate signals on T2 weighted MR images and strong, homogeneous contrast enhancement on enhanced T1 weighted MR images. ${ }^{1}$ The lesion may show increased activity on PET. On histopathologic examination, the hallmark of Rosai-Dorfman disease is emperipolesis, in which lymphocytes and other inflammatory cells reside undisturbed within the cytoplasm of the histiocytes. However, this is found much less commonly in the extranodal lesions. The histiocytes in RDD are immunopositive for S-100 protein, CD68 and negative for CD1a. ${ }^{1}$ The clinical and microscopic manifestation of RDS should be differentiated from malignant lymphoreticular neoplasias such as Hodgkin's disease and monocytic leukemia, Langerhans cell histiocytosis, rhinoscleroma, tuberculosis and Wegener's granulomatosis.
Prominent emperipolesis, polymorphous cell population, absence of grooved nuclei of histiocytes, absence of eosinophils, absence of Reed Sternberg cells and absence of epitheloid granulomas aid in the diagnosis of RDD. ${ }^{12}$

The prognosis of RDD is variable. Most patients have an indolent course that is characterized by exacerbations and remissions. Extranodal RDD tends to be chronic and relapsing, and two thirds of patients with sinonasal RDD have persistent or progressive disease which may pose a diagnostic challenge both for the clinicians and radiologists. In general, extranodal involvement does not determine a more aggressive character or poor outcome; however, generalized lymphadenopathy, extranodal involvement of multiple organs (kidney, lungs and liver) and immunologic alterations lead to a poor prognosis. Even though it is considered a benign disease, fatalities could occur due to cellular infiltrate. Very rarely, RDD progression to malignant lymphoma and to amyloidosis have been documented.

No specific treatment has been advocated for this essentially self-limiting disease, which has a good prognosis. ${ }^{3}$ Treatment is necessary only when the disorder becomes symptomatic. There is no specific treatment for RDS. The treatment modes include corticosteroids, chemotherapy with a combination of vinca alkaloids and alkylating agents, low dose interferon, antibiotics, radiation therapy, imatinib mesylate and surgical treatment with partial or total resection. ${ }^{6}$

In our patient, the disease involved the left nasal cavity and left maxillary sinus with erosion of the posterolateral wall and orbital encroachment. There was no evidence of cervical lymphadenopathy. There was no concomitant involvement of any other extranodal site. Endoscopic excision of the mass was done and the patient was under regular follow-up. Recurrence was excised by laserization with KTP/532 laser followed by oral corticosteroid therapy (60 mg/day for 1 week and then tapered over the next 3 weeks). Our patient has been advised periodic follow-ups.

In a case report by SS Bist et al, the nasal mass was excised endoscopically and the patient was treated with oral prednisolone $60 \mathrm{mg} /$ day for a period of 8 weeks and then tapered off. ${ }^{6}$ In a case report by Yu-Ting Huang et al surgical resection of sinonasal RDD was done. One year later, the patient had an intracranial extra-axial durabased mass in the right frontal region in addition to the residual infiltrative sinonasal mass. She underwent surgical resection of the frontal intracranial mass. For the treatment of recurrent sinonasal RDD, she received radiotherapy (4200 cGy/21 fractions). ${ }^{1}$ In a report by Matthias Hagemann et al in one patient, endonasal tumor debulking was done and because steroids had not achieved any improvement of the nasal symptoms, 
fractionated low-dose radiotherapy was given in the region of the paranasal sinuses with a total dose of $20 \mathrm{~Gy}$. In the second patient, functional endoscopic sinus surgery was performed and postopertive steroids were given. ${ }^{10}$ In a report by L Gupta et al functional endoscopic sinus surgery was done and diagnostic nasal endoscopy done postoperatively did not reveal any polyps or residual disease. ${ }^{3}$ In a case report by Belcadhi $\mathrm{M}$ et al $\mathrm{CO}_{2}$ laser excision was used to clear the nasal obstruction with a satisfactory result. ${ }^{13}$

\section{CONCLUSION}

Sinonasal Rosai-Dorfman disease is a rare condition presenting with symptoms of sinusitis. As the clinical picture could be variable, the diagnosis may be easily overlooked by both the ENT surgeon and the pathologist. Although, cervical lymphadenopathy is the most common presentation encountered, extranodal presentation in the head and neck occurs in about $40 \%$ of cases. Hence, the otolaryngologist must be familiar with the clinical manifestations, diagnosis and management of Rosai-Dorfman disease.

\section{ACKNOWLEDGMENT}

The authors gratefully acknowledge the contribution of Dr Ashok Parameswaran, Consultant Pathologist for his contribution on the histopathology and immunohistochemistry of Rosai-Dorfman disease.

\section{REFERENCES}

1. Yu-Ting Huang, Shu-Hang Ng, Sheung-Fat Ko, et al. Extranodal Rosai-Dorfman disease with paranasal sinuses and intracranial involvement: a case report. Chin J Radiol 2009;34:191-196.

2. Sanli A, Eken M, Oktay ZA, et al. Rosai-Dorfman disease: sinus histiocytosis with massive lymphadenopathy. BMJ Case Rep. doi: 10.1136/bcr.11.2008.1251.

3. Gupta L, Batra K, Motwani G. A rare case of RosaiDorfman disease of the paranasal sinuses. Indian Journal of Otolaryngology and Head and Neck Surgery 2005 OctDec;57(4):352-354.

4. Petzold A, Thom M, Powell M, et al. Relapsing intracranial Rosai-Dorfman disease. J Neurol Neurosurg Psychiatry 2001; 71:538-541.

5. Wang E, Anzai Y, Paulino A, et al. Rosai-Dorfman disease presenting with isolated bilateral orbital masses: report of two cases. Am J Neuroradiol 2001 Aug;22:1386-1388.

6. Bist SS, Bisht M, Varshney S, et al. Rosai-Dorfman syndrome with extranodal manifestation. JAPI 2007 Jun;55:445-447.

7. Rosai J, Dorfman RF. Sinus histiocytosis with massive lymphadenopathy: a pseudolymphomatous benign disorder. Cancer 1972;30:1174-1188.

8. Barge DVL, Salzman KL, Harnsberger HR, et al. Sinus histiocytosis with massive lymphadenopathy (Rosai-Dorfman disease): Imaging manifestations in the Head and Neck. Neuroradiology/Head and Neck Imaging 2008 Dec;191(6):299-306.

9. Shiran MS, Tan GC, Kenali MS, et al. Multifocal nodal and extranodal Rosai-Dorfman disease initially diagnosed as histiocytic lymphoma. Malaysian J Pathol 2008;30(1):63-65.

10. Hagemann M, Zbären P, Stauffer E, et al. Nasal and paranasal sinus manifestation of Rosai-Dorfman disease. Rhinology 2005;43:229-232.

11. Kharrat S, Sahtout S, Oukhai M, et al. Multifocal RosaiDorfman disease: a case report. Fr ORL 2008;94:395-398.

12. Jena M. Diagnosis of Rosai-Dorfman disease by fine needle aspiration cytology in a case with cervical lymphadenopathy and nasal mass. Online J Health Allied Scs 2011;10(2):22.

13. Belcadhi M, Bellakhdhar M, Sriha B, et al. Rosai-Dorfman disease of the nasal cavities: $\mathrm{A} \mathrm{CO}_{2}$ laser excision. Am J Rhinol Allergy 2010 Jan-Feb;24(1):91-93. 